\title{
Third Degree Volterra Kernel for Newborn Cry Estimation
}

\author{
Gibran Etcheverry ${ }^{1}$, Efraín López-Damian ${ }^{2}$, and Carlos A. Reyes-García ${ }^{3}$ \\ 1 DIFUS-USON, Encinas y Rosales S/N, 83000, Hermosillo, Mexico \\ gibran.etcheve@difus.uson.mx \\ 2 FIME-CIIDIT-UANL, Mechatronics Department, PIIT, 66600, Apodaca, \\ Nuevo León, Mexico \\ efrain.lopezdm@uanl.edu.mx \\ 3 INAOE, Department of Computer Science, Enrique Erro 1, 72840, \\ Tonantzintla, Mexico \\ kargaxxi@ccc.inaoep.mx
}

\begin{abstract}
Newborn cry analysis is a difficult task due to its nonstationary nature, combined to the presence of nonlinear behavior as well. Therefore, an adaptive hereditary optimization algorithm is implemented in order to avoid the use of windowing nor overlapping to capture the transient signal behavior. Identification of the linear part of this particular time series is carried out by employing an Autorregresive Moving Average (ARMA) structure; then, the resultant estimation error is approched by a Nonlinear Autorregresive Moving Average (NARMA) model, which realizes a Volterra cubic kernel by means of a bilinear homogeneous structure in order to capture burst behavior. Normal, deaf, asfixia, pain, and uncommon newborn cries are inspected for differentation.
\end{abstract}

\section{Introduction}

The newborn cry represents the heart-breathing coordination activity with the muscular larynx. The cry is an acoustics event that contains information about the central nerves system functioning as well. It is one of the most important natural ways of communication that a newborn has to interact with his/her environment; through crying, the newborn babies express their needs and basic feelings like hungry or pain. Crying is the only non visual option that neonatologists or pediatricians have for disease or malaise recognition.

The Golub [1] crying model is divided into four parts. The first part is the glottal system that is in charge to develop the pressure under the glottis necessary to drive the vocal folds. The second part is the source of sound located at the larynx. This sound source can be described mathematically, in frequency domain, as a periodic source or like noisy source. All these sources can work in an isolated or simultaneous way. Both acoustics sources are originated in the vocal folds. The periodic source is produced by the vocal folds vibration. The noise is seen as a turbulence and is the result of the forced air passing through a small opening 
between the vocal folds from the lungs. The third part is composed by the vocal and nasal tract in the larynx, these elements act like an acoustics filter where its transfer function characteristics change due to their form and length. The four part is the radiation that describes the filtering of sound between the infant mouth and a microphone located at a certain distance to obtain this acoustic signal [2].

Nonstationary signals are those which present a time variable change of their statistical properties and frequency content [3]. For instance, we can find them in the areas of geology on seismic events [4] and of biomedicine over Heart Rate Variability (HVR) studies [5], electroencephalogram (EEG) characterization [6], newborn cry [2] and children speech analysis [7, among others.

In the case of crying, the first studies started back in 1838 with Gardiner, using music notes and in 1855 Darwing studied the local anatomy and physiology of speech development and its relation with the emotion expression. Babies cannot satisfy their necessities by themselves, they completely depend on the attentions of adults. The cry is the natural way of communication to express their different emotional and physiological states.

The widespread presence of these kind of signals has required to study them deeply in order to understand their behavior; for example, some of them present an intermittent sequence of frequency content and chaotic behavior in time.

Newborn cry classification is based on linear features extraction in the area of Artificial Intelligence (AI) 2. In the case of speech, Linear Predictive Coefficients (LPC) analysis has been carried out followed by a quadratic degree Volterra filtering of the obtained residual for speech coding [8] 9], analysis and synthesis [10.

In this work, the hereditary computation of the signal correlation function, gives us the possibility to determine the time variyng parameters between windows of time automatically without windowing nor overlapping. This adaptive task is carried out by an autorregressive moving average (ARMA) model[11] in order to approach the linear part of five kinds of newborn cries: normal, deaf, asfixia, pain, and uncommon. Thus, the resulting estimation error is projected onto a cubic degree Volterra kernel by means of a particular bilinear structure [12. A good estimation performance of newborn cry signals is obtained due to burst modeling by nonlinear approximation.

\section{Adaptive Hereditary Computation}

Adaptive hereditary computation has its origin in the area of systems identification by employing an ARMA model, in order to fit a stochastic realization to some measured output $y_{\tau}, \tau=1, \ldots, t$ of an unknown system. The one-step ahead predictor therefore implemented, estimates the model coefficients without using a non linear optimization technique, as it is the case for gradient based or Gauss-Newton techniques [11. Instead of it, recomputation of $\tau=1, \ldots, T$ past samples is developed by using the parameters obtained at time $t$; hence, the correlation function is time variyng and up to date, avoiding in this way 
calculation errors due to incomplete correlation terms obtained with standard estimation methods.

\subsection{ARMA Form}

The one-step ahead predictor in ARMA form is written as follows:

$$
\hat{y}_{t}=\sum_{i=1}^{n} a_{i} \hat{y}_{t-i}+\sum_{j=1}^{n} b_{i} \tilde{y}_{t-j}
$$

where $\tilde{y}_{t}=y_{t}-\hat{y}_{t}$ and $a_{i}, b_{i}$ are the autorregresive and input coefficients, respectively.

\subsection{Hereditary Computation}

As it was stated in the begining of this section, the transient optimization approach can be developed to the price of hereditary computation of the model coefficients, presenting a linear $t$-growing memory of size $n t$, with $n$ as the system dimension or delay. Hence, the ARMA form having these characteristics is written as:

$$
\hat{y}_{\tau}^{t}=\sum_{i=1}^{n} a_{i}^{t} \hat{y}_{\tau-i}^{t-i}+b_{i}^{t} \tilde{y}_{\tau-i}^{t-i}, \quad \forall \tau=1, \ldots, t .
$$

In order to obtain the model parameters, it is necessary to employ an evaluation criterium of how well (2) performs; this criterium consists on minimizing the mean square error $(\mathrm{MSE})$ between the time series $y_{t}$ and the predictor $\hat{y}_{\tau}^{t}$ :

$$
J_{T}^{t}=E_{T}^{t}\left[\left(y_{\tau}-\hat{y}_{\tau}^{t}\right)^{2}\right]=\frac{1}{T} \sum_{\tau=t-T+1}^{t}\left(y_{\tau}-\hat{y}_{\tau}^{t}\right)^{2} \quad \tau=1, \ldots, T .
$$

where $T$ is the time horizon of hereditary computation or re-computation of the estimated samples. This means that the estimator adapts its horizon every $T$ samples to get the time-variyng parameters that characterize the signal of interest.

Derivating (3) with respect to the model parameters and separating terms to each side of the equality, leads us to the normal equations, see [13], which contain the time-varying correlation and intercorrelation terms:

$$
\left[\begin{array}{lll}
\sum_{\tau=t-T+1}^{t} \hat{y}_{\tau-i}^{t-i} \hat{y}_{\tau-i}^{t-i} & \sum_{\tau-t-T+1}^{t} & \hat{y}_{\tau-i}^{t-i} \tilde{y}_{\tau-i}^{t-i} \\
\sum_{\tau=t-T+1}^{t} \tilde{y}_{\tau-i}^{t-i} \hat{y}_{\tau-i}^{t-i} & \sum_{\tau=t-T+1}^{t}=\tilde{y}_{\tau-i}^{t-i} \tilde{y}_{\tau-i}^{t-i}
\end{array}\right]\left[\begin{array}{c}
a_{i}^{t} \\
b_{i}^{t}
\end{array}\right]=\left[\begin{array}{c}
\sum_{\tau-t-T+1}^{t} y_{\tau} \hat{y}_{\tau-i}^{t-i} \\
\sum_{\tau=t-T+1}^{t=t} y_{\tau} \tilde{y}_{\tau-i}^{t-i}
\end{array}\right] i=1 \ldots n .
$$

Once the aforementioned model parameters are calculated, it is possible to build the system transfer function and obtain the estimated output time series or the estimated system impulse response. 


\section{Nonstationary Behavior}

A time series is considered nonstationary when there is a considerable part of the power spectra in the low frequencies, since the corresponding mode has very few oscillations during the observation time. Besides this, if the signal alternates between periodic and irregular behavior, it presents a nonlinear phenomena called intermittency. These caotic phases can be long or in the form of bursts [14. The newborn cry presents both characteristics, a periodic behavior with and important spectral component in the low frequencies (pitch of $400-600 \mathrm{~Hz}$ ) combined with short intermittencies or bursts, see Fig. 1 .
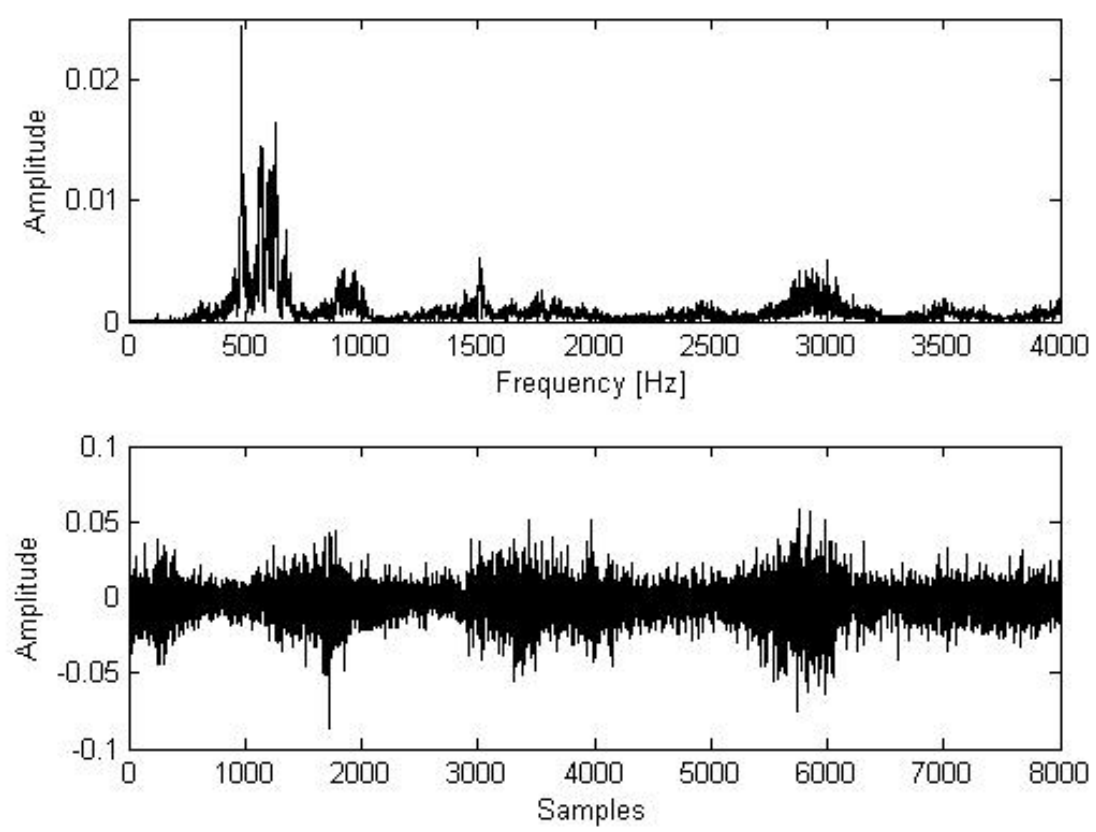

Fig. 1. Normal newborn cry spectra (up) and linear estimation error presenting bursts (bottom)

Figure 2 shows a different spectral pattern distribution as well as very frequent jumps on the estimation error obtained by using (2).

\subsection{Cubic Volterra Estimation}

The error $\tilde{y}_{t}^{1}$ resulting of substracting the ARMA estimator (2) from the measured time series $y_{t}$, can be approximated by a cubic Volterra kernel $K_{3}$ of the form:

$$
\hat{y}_{t}=y_{t}-\hat{y}_{\tau}^{t}=\sum_{\tau_{1}=1}^{t} \sum_{\tau_{2}=1}^{\tau_{1}} \sum_{\tau_{3}=1}^{\tau_{2}} K_{3}\left(t, \tau_{1}, \tau_{2}\right) \tilde{y}_{\tau_{1}} \tilde{y}_{\tau_{2}} \tilde{y}_{\tau_{3}}
$$



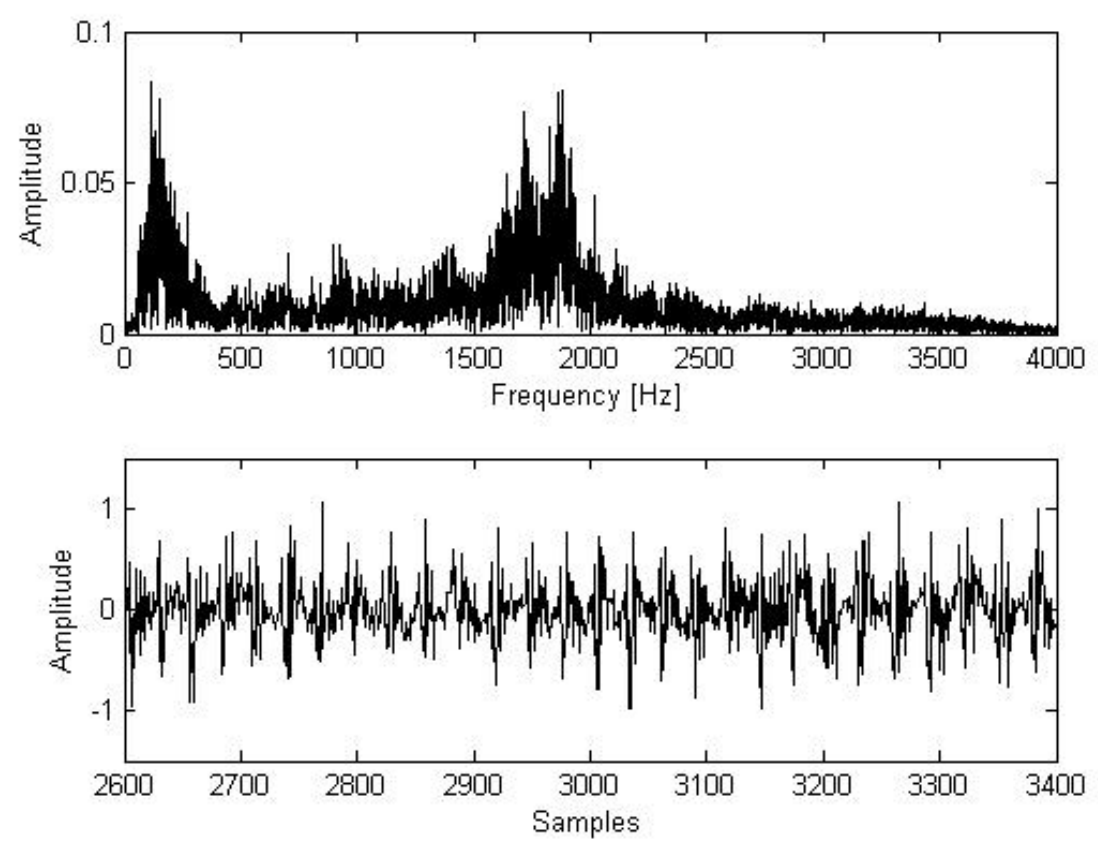

Fig. 2. Uncommon newborn cry spectra (up) and linear estimation error presenting glitches (bottom)

\subsection{Bilinear Structure}

It is a well known fact that a disadvantage of using a Volterra series for modeling nonlinear events is the curse of dimensionality, which makes the number of coefficients employed to grow fast with prediction horizon [8. In the view of overcoming this feature, a bilinear homogeneous structure can generate separated Volterra series kernels recursively, yielding few parameters and allowing to choose the nonlinear degree of estimation [15].

Proposition: The homogeneous bilinear system that realizes the cubic Volterra kernel $K_{3}\left(t, \tau_{1}, \tau_{2}\right)$ is the following:

$$
\begin{gathered}
x_{t}^{1}=A_{t}^{1} x_{t-1}^{1}+B_{t} \tilde{y}_{t} \\
x_{t}^{2}=A_{t}^{2} x_{t-1}^{2}+D_{t}^{2} x_{t}^{1} \tilde{y}_{t} \\
x_{t}^{3}=A_{t}^{3} x_{t-1}^{3}+D_{t}^{3} x_{t}^{2} \tilde{y}_{t} \\
\hat{y}_{t}^{3}=C_{t} x_{t}^{3}
\end{gathered}
$$

with

$$
B_{t}^{1}=\left[b_{n_{1}, t}^{1} \ldots b_{1, t}^{1}\right]^{T}
$$




$$
\begin{aligned}
& D_{t}^{k}=\left[\begin{array}{cccc}
d_{n_{k}, n_{k-1}, t}^{k} & \ldots & \ldots & d_{n_{k}, 1, t}^{k} \\
\vdots & \vdots & \vdots & \vdots \\
d_{2, n_{k-1}, t}^{k} & \ldots & \ldots & d_{2,1, t}^{k} \\
0 & \ldots & 0 & 1
\end{array}\right] ; \forall k=2 \ldots d \\
& A_{t}^{k}=\left[\begin{array}{ccccc}
0 & \ldots & \ldots & 0 & a_{n_{k}, t}^{k} \\
1 & \ddots & & \vdots & \vdots \\
0 & \ddots & \ddots & \vdots & \vdots \\
\vdots & \ddots & \ddots & 0 & \vdots \\
0 & \ldots & 0 & 1 & a_{1, t}^{k}
\end{array}\right] ; \forall k=1 \ldots d \\
& C=\left[\begin{array}{llll}
0 & \ldots & 0 & 1
\end{array}\right]
\end{aligned}
$$

where $x_{t}^{k} \in \Re^{n_{3}}$ and $A_{t}^{k} \in \Re^{n_{3} \times n_{3}}, \forall k=1 \ldots 3, D_{t}^{k} \in \Re^{n_{3} \times n_{2}}, \forall k=2,3, C_{t} \in \Re^{n_{3}}$ and $B_{t} \in \Re^{n_{1}}$.

Proof: Every state $x_{t}^{k}$, for $k=1 \ldots 3$ can be considered as a linear system output with input $D_{t}^{k} x_{t}^{k-1} \tilde{y}_{t}$. Thus, we have:

$$
\begin{gathered}
x_{t}^{1}=\sum_{\tau=1}^{t} \phi^{1}(t, \tau) B_{\tau} \tilde{y}_{\tau} \\
x_{t}^{2}=\sum_{\tau=1}^{t} \phi^{2}(t, \tau) D_{\tau}^{2} x_{\tau}^{1} \tilde{y}_{\tau} \\
x_{t}^{3}=\sum_{\tau=1}^{t} \phi^{3}(t, \tau) D_{\tau}^{3} x_{\tau}^{2} \tilde{y}_{\tau} \\
\hat{y}_{t}^{3}=C_{t} x_{t}^{3}
\end{gathered}
$$

where $\phi^{k}(t, \tau)=A_{t}^{k} \phi^{k}(t-1, \tau)$ is the transition matrix and $K_{3}\left(t, \tau_{1}, \tau_{2}\right)=$ $\left.\left.C_{t} \phi^{3}(t, 1)\left(\phi^{3}\left(\tau_{1}, 1\right)^{-1}\right) D_{\tau_{1}}^{3} \phi^{2}\left(\tau_{1}, 1\right)\left(\phi\left(\tau_{2}, 1\right)\right)^{-1}\right) D_{\tau_{2}}^{2} \phi^{1}\left(\tau_{2}, 1\right)\left(\phi\left(\tau_{3}, 1\right)\right)^{-1}\right) B_{\tau_{1}}$.

In this work this kind of structure is used, but in order to be able to adapt the hereditary computation to it, the matrix canonical form in (6) has been developped to end up with a NARMA expression of the form:

$$
\begin{gathered}
y_{t}^{1}=\sum_{i=1}^{n_{1}} a_{i, t}^{1} y_{t-i}^{1}+\sum_{i=1}^{n_{1}} b_{i, t}^{1} \tilde{y}_{t-i+1} \\
y_{t}^{2}=\sum_{i=1}^{n_{2}} a_{i, t}^{2} y_{t-i}^{2}+y_{t}^{1} \tilde{y}_{t}+\sum_{i=2}^{n_{2}} \sum_{j=1}^{n_{1}} d_{i, j, t}^{2} y_{t-i-j+2}^{1} \tilde{y}_{t-i+1} \\
y_{t}^{3}=\sum_{i=1}^{n_{3}} a_{i, t}^{3} y_{t-i}^{3}+y_{t}^{2} \tilde{y}_{t}+\sum_{i=2}^{n_{3}} \sum_{j=1}^{n_{2}} d_{i, j, t}^{3} y_{t-i-j+2}^{2} \tilde{y}_{t-i+1}
\end{gathered}
$$

The expresion for any nonlinear degree of (6) and derivation of (8) can be found in $[12]$.

\subsection{Algorithm}

Resuming the last two sections, the algorithm used in this work is the following:

1. Estimate the linear part of the signal by using (2), taking as optimization criterion (3). 
2. Obtain and approach the resulting error on (5) by employing the estimator (8) at time $t$ :

$$
\begin{gathered}
\hat{y}_{\tau}^{1, t}=\sum_{i=1}^{n_{1}} a_{i, t}^{1} \hat{y}_{\tau-i}^{1, t-i}+\sum_{i=1}^{n_{1}} b_{i, t}^{1} \tilde{y}_{t-i+1} \\
\ldots \\
\hat{y}_{\tau}^{3, t}=\sum_{i=1}^{n_{3}} a_{i, t}^{3} \hat{y}_{\tau-i}^{3, t-i}+\hat{y}_{\tau}^{2, t} \tilde{y}_{t}+\sum_{i=2}^{n_{3}} \sum_{j=1}^{n_{2}} d_{i, j, t}^{3} \hat{y}_{\tau-i-j+2}^{2, t-i-j+2} \tilde{y}_{t-i+1}
\end{gathered}
$$

where $\hat{y}_{\tau}^{1, t-1}$ and $\hat{y}_{\tau}^{3, t-1}, \forall \tau=1, \ldots, t-1$ were obtained by minimizing the criterion:

$$
J_{T}^{t-1}=E_{T}^{t-1}\left[\left(\tilde{y}_{t}-\hat{y}_{*}^{3, t-1}\right)^{2}\right]=\frac{1}{T+t-1} \sum_{\tau=T}^{t-1}\left(\tilde{y}_{t}-\hat{y}_{*}^{3, t-1}\right)^{2}
$$

and $T$ is the time horizon of hereditary computation as in (3).

3. Obtain the kernel coefficients.

\section{Results}

This work has analyzed 10 recordings of 5 different kinds of newborn cries each: normal, deaf, asfixia, pain, and uncommon. These are one second recordings sampled at $F_{s}=8000 \mathrm{~Hz}$ that have been treated in segments of time horizon $T=20 \mathrm{~ms}$. It is important to mention that there is no overlapping between segments nor windowing to carry out the analysis.

The order or dimension used for the linear estimator was of $n=10$, considering the experience on speech and newborn cry analysis [2].

After doing some testing, it was realized that the cubic kernel yields a good estimation from the beforehand mentioned error by using a dimension $n=1$, leaving us with the expression (9) reduced to:

$$
\hat{y}_{\tau}^{3, t}=a_{1, t}^{3} \hat{y}_{\tau-1}^{3, t-1}+a_{1, t}^{2} \hat{y}_{\tau-1}^{2, t-1} \tilde{y}+a_{1, t}^{1} \hat{y}_{\tau-1}^{1, t-1} \tilde{y}^{2}+b_{1, t}^{1} \tilde{y}^{3}
$$

The algorithm quality is assessed after estimation by the criterium:

$$
R=\left(1-\frac{\sum_{t=1}^{N}\left|y_{t}-\hat{y}_{t}\right|^{2}}{\sum_{t=1}^{N}\left|y_{t}\right|^{2}}\right)
$$

with $N$ the number of samples.

Table 1 shows that in a few cases using linear estimation suffices to describe a newborn cry behavior, whereas in most of them it is not enough to describe this kind of signal. This is an even more important characteristic in cases where the linear signal estimation performance is below 80 percent, as it is the case mainly for deaf newborn cry analysis. Pain crying was obtained by light stimulation of the infants and uncommon crying analysis has been included to show that linear signal estimation is not enough when the crying behavior presents abrupt changes, see Fig 2 . 
Table 1. Linear ARMA and Linear+Cubic Volterra Series Fit

\begin{tabular}{|c|c|c|c|c|c|c|c|c|c|}
\hline \multicolumn{2}{|c|}{ Normal } & \multicolumn{2}{c|}{ Deaf } & \multicolumn{2}{c|}{ Asfixia } & \multicolumn{2}{c|}{ Pain } & \multicolumn{2}{c|}{ Uncommon } \\
\hline Lin & Lin+Cub & Lin & Lin+Cub & Lin & Lin+Cub & Lin & Lin+Cub & Lin & Lin+Cub \\
\hline 82.75 & 99.23 & 67.91 & 97.66 & 97.30 & - & 90.18 & 99.51 & 85.70 & 97.89 \\
95.62 & 99.64 & 70.79 & 96.01 & 55.00 & 89.62 & 84.84 & 96.85 & 87.48 & 99.15 \\
90.10 & 98.95 & 72.47 & 95.76 & 89.33 & 98.86 & 86.18 & 99.19 & 88.44 & 99.08 \\
90.83 & 99 & 75.08 & 96.31 & 96.83 & - & 88.37 & 98.73 & 97.20 & - \\
97.29 & - & 73.51 & 97.81 & 78.07 & 99.76 & 77.53 & 96.10 & 91.43 & 99.33 \\
82.65 & 98.98 & 65.93 & 95.45 & 90.08 & 99.74 & 77.77 & 96.74 & 97.26 & - \\
91.54 & 99.59 & 59.05 & 97.54 & 90.08 & 98.94 & 78.02 & 97.31 & 97.60 & - \\
92.70 & 99.56 & 82.28 & 98.97 & 92.07 & 96.46 & 77.65 & 97.27 & 83.35 & 97.45 \\
69.14 & 98.77 & 77.12 & 97.88 & 98.15 & - & 85.03 & 97.85 & 87.01 & 98.83 \\
87.75 & 99.28 & 77.12 & 96.98 & 99.06 & - & 79.82 & 97.77 & 71.96 & 95.72 \\
\hline
\end{tabular}
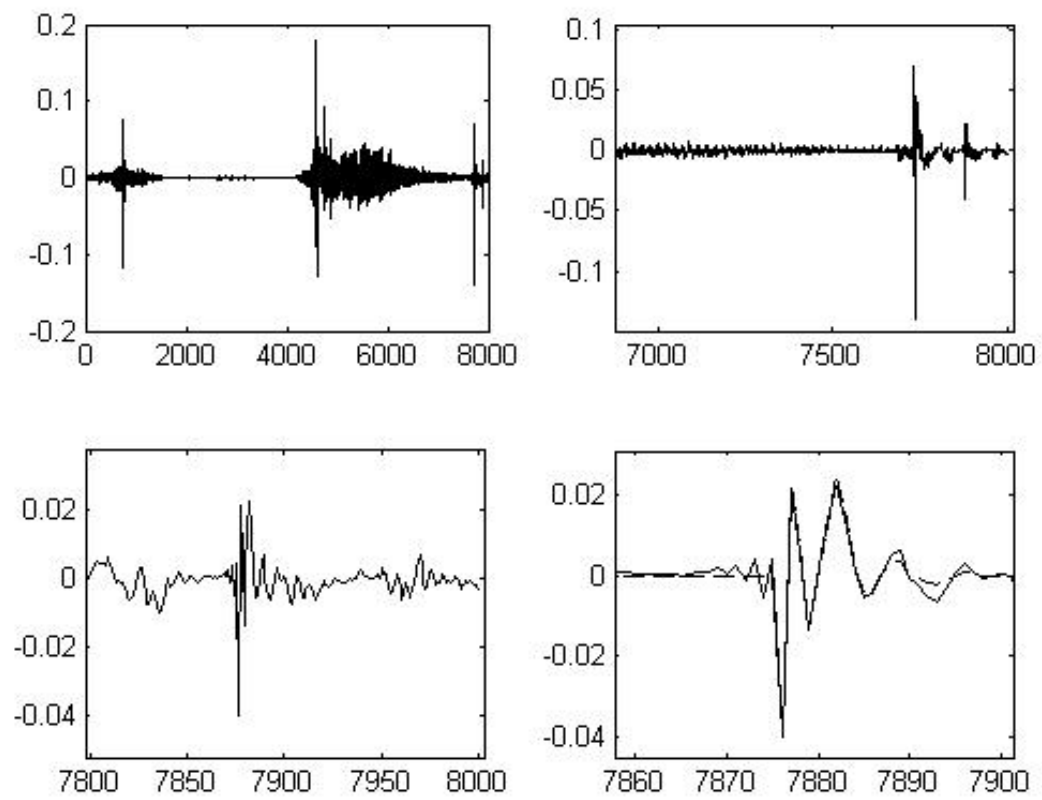

Fig. 3. Asfixia newborn cry burst close up images and estimation (bottom right, broken line)

\section{Conclusions}

This work presents as main contributions:

- The study of intermittencies present within newborn cries by using cubic Volterra analysis, which to the authors knowledge, it hasn't been implemented until now. 
- The non use of overlapping nor windowing to compensate the nonstationary newborn cry behavior; the signal was just directly analized on segments of $20 \mathrm{~ms}$ due to hereditary computation.

- The possibility of obtaining nonlinear characteristics from newborn cries, which will be useful to the community of pattern recognition and classification.

Once the Volterra kernels are realized, the estimator is able to recontruct the time series behavior based on the obtained error. There are some recent works on estimation of chaotic time series, see [16] and [17, but they employ different Volterra kernel realizations. An interesting comparison of performance can be developped with optimization methods like the one shown in [18; even though, these models are neither autorregresive nor transient optimized, see [19] and [11] respectively.

Given the close relation between the acoustics aspects of the crying and the anatomical and physiological characteristics of the newborn, the mathematical theory of signal processing plays an important part for the fundamental research about medical applications like diagnosis based on crying. Estimation based on hereditary and Volterra techniques allows to obtain a more accurate model of the crying signal, and therefore it can make certainly these kind of applications easier.

\section{References}

1. Golub, H., Corwin, M.: Infant cry: a clue to diagnosis. Pediatrics 69, 197-201 (1982)

2. Reyes-García, C., Cano-Ortiz, S.: Fundamentos Téoricos y Prácticos del Análisis de Llanto Infantil. Inaoe-Conacyt (2009)

3. Priestley, M.: Nonlinear and Nonstationary Time Series Analysis. Academic Press, London (1988)

4. Ezekiel, S., et al.: Seismic signal analysis using correlation dimension. In: Proc. in Applied Informatics (2003)

5. Lee, F.A., Nehorai, A.: Adaptive power spectrum estimation algorithm for heart rate variability analysis. Proc. of the IEEE, 273-276 (1992)

6. Karjalainen, P.: Estimation Theoretical Background of Root Tracking Algorithms with Applications to EEG. University of Kuopio Department of Applied Physics, Report Series (1996) ISSN 0788-4672

7. Potaminos, A., Narayanan, S.: A review of the acoustic and linguistic properties of children's speech. In: IEEE 9th Workshop on Multimedia Signal Processing, MMSP, pp. 22-25 (2007)

8. Thyssen, J., Nielsen, H., Hansen, S.: Nonlinear short-term prediction in speech coding. IEEE Proceedings I, 185-188 (1994)

9. Alipoor, G., Savoji, M.: Speech coding using nonlinear prediction based on volterra series expansion. In: SPECOM, pp. 367-370 (2006)

10. Schnell, K., Lacroix, A.: Voiced excitation models for speech production based on time variable volterra systems. In: NOLISP, pp. 184-187 (2005)

11. Monin, A., Salut, G.: Arma lattice identification: A new hereditary algorithm. IEEE Trans. on Signal Processing 44(2), 360-370 (1996) 
12. Etcheverry, G., Suleiman, W., Monin, A.: Quadratic system identification by hereditary approach. In: ICASSP, vol. III, pp. 129-132 (2006)

13. Ljung, L.: System identification: theory for the user. Prentice-Hall, Englewood Cliffs (1999)

14. Kantz, H., Schreiber, T.: Nonlinear Time Series Analysis. Cambridge University Press, Cambridge (1997)

15. Rugh, J.: Nonlinear System Theory: The Volterra/Wiener Approach. Wiley, Chichester (1980)

16. Li, C., Yu, J.: Volterra-tls method for chaotic time series prediction. Proc. of the IEEE, 48-51 (2008)

17. Wang, H., Gu, H.: Prediction of chaotic time series based on neural network with legendre polynomials. LNCS, vol. 5551, pp. 836-843. Springer, Heidelberg (2009)

18. Jirong, G., Xianwei, C., Jieming, Z.: An algorithm of predictions for chaotic time series based on volterra filter. In: ISECS, Proc. of the IEEE Computer Society, vol. 2, pp. 205-208 (2009)

19. Monin, A., Salut, G.: I.i.r volterra filtering with application to bilinear systems. IEEE Trans. on Signal Processing 44(9), 2209-2221 (1996) 